\title{
Discovery of pulsation in the Am Star HD 102480
}

\author{
S. Joshi ${ }^{1}$, V. Girish ${ }^{2}$, R. Sagar ${ }^{1}$, D. W. Kurtz ${ }^{3}$, P. Martinez ${ }^{4}$ and S. Seetha ${ }^{2}$ \\ ${ }^{1}$ State Observatory, Manora Peak, Naini Tal-263 129, India, santosh@upso.ernet.in \\ ${ }^{2}$ ISRO Satellite Centre, Air Port Road Bangalore-560 034, India \\ ${ }^{3}$ Centre for Astrophysics, University of Central Lancashire, Preston PR1 2HE UK \\ ${ }^{4}$ South African Astronomical Observatory, P. O. Box 9, Observatory 7935, \\ South Africa
}

We report the discovery of pulsation in the marginally chemically peculiar Am star HD 102480. This star was discovered during the "Naini Tal-Cape Survey for Pulsations in Chemically Peculiar Stars" (Martinez et al. 2001). HD $102480\left(V=8.45, \alpha_{2000}=11^{h} 47^{m} 52^{s}, \delta_{2000}=53^{\circ} 00^{\prime} 54^{\prime \prime}\right)$ is a cool Am star of spectral type Am (F2/F4/F4) (Abt 1984), where the types in brackets are the $\mathrm{Ca}$ II K-line type, Balmer line type and metal line type, respectively. It has Strömgren indices corresponding to the marginally enhanced metallicity indicated by its spectral type, viz. $b-y=0.211, m_{1}=0.204$, $c_{1}=0.732$ (Olsen 1983); $\delta m_{1}=-0.025$ and $\delta c_{1}=0.087$ using Crawford's calibrations of the Strömgren indices for A stars (Crawford 1979).

On the basis of these peculiar colours, we decided to search for pulsation in HD 102480 on the night of 19 January 2000 (HJD 2451563). The data were acquired as continuous 10-s integrations through a Johnson $B$ filter with a high-speed photometer attached to the 104-cm Sampurnand telescope of State Observatory, Naini Tal (Gupta et al. 2001). A variation of 0.05 mag was observed in the star on this night, indicative of $\delta$ Sct pulsation.

Fig. 1 shows the discovery light curve obtained on JD 2451563, and the confirming light curve obtained on night JD 2451598 using the same telescope/instrument combination. The light curves are corrected for coincidence counting losses, sky background and mean atmospheric extinction. From the light curves it can be seen that the amplitude of the pulsation is modulated with a maximum peak-to-peak variation of $\approx 0.08$ mag.

The corrected data were Fourier analysed to identify the dominant periodicities using a fast algorithm based on Deeming's (1975) Discrete Fourier Transform (DFT). The amplitude spectra show two strong peaks at $0.10 \pm 0.02 \mathrm{mHz}$ $(2.78 \pm 0.56 \mathrm{hr})$ and $0.19 \pm 0.02 \mathrm{mHz}(1.46 \pm 0.15 \mathrm{hr})$. On the basis of the periods, the shape of the light curve and the amplitude variation during our 

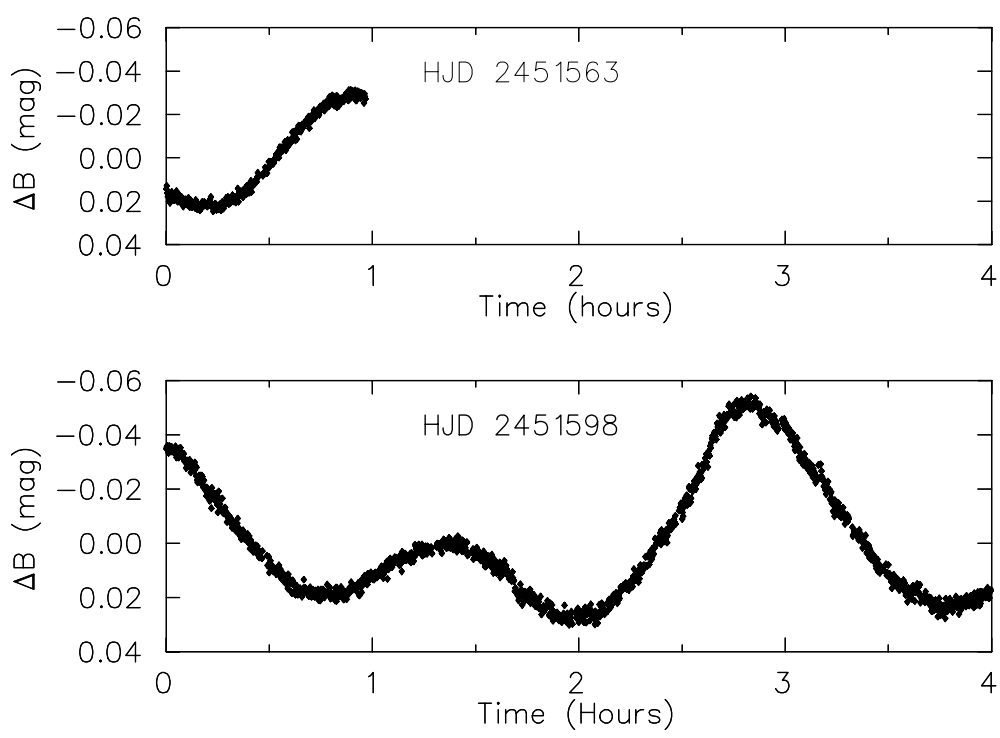

Figure 1: The light curves of HD 102480 on HJD 2451563 and HJD 2451598.

observations, we announce that HD 102480 is a new $\delta$ Scuti star with marginal Am peculiarities (indicated by both its spectral type and Strömgren indices).

\section{References}

Abt, H. A. 1984, ApJ 285, 247

Crawford, D. 1979, AJ 84, 1858

Deeming, T. J. 1975, Ap\&SS 36, 137

Gupta, S. K., Sagar, R., Joshi, S., Ashoka, B. N., Babu, V. C, Seetha, S., Girish, V. 2001, BASI 29, 479

Martinez, P., Kurtz, D. W., Ashoka, B. N., Chaubey, U. S., Girish, V., Gupta, S. K., Joshi, S., Kasturirangan, K., Sagar, R., Seetha S. 2001, A\&A 371, 1048

Olsen, E.H. 1983 A\&AS 54, 55 